\title{
Flow structure over square bars at intermediate submergence: Large Eddy Simulation study of bar spacing effect
}

\author{
Thorsten STOESSER ${ }^{1}$ and Vladimir I. NIKORA ${ }^{2}$ \\ ${ }^{1}$ School of Civil and Environmental Engineering, \\ Georgia Institute of Technology, Atlanta, USA \\ e-mail: thorsten@ce.gatech.edu (corresponding author) \\ ${ }^{2}$ Kings College, School of Engineering, University of Aberdeen \\ Aberdeen, Scotland, United Kingdom \\ e-mail: v.nikora@abdn.ac.uk
}

\begin{abstract}
Turbulent open-channel flow over 2D roughness elements is investigated numerically by Large Eddy Simulation (LES). The flow over square bars for two roughness regimes (k-type roughness and transitional roughness between d-type and k-type) at a relative submergence of $H / k=6.5$ is considered, where $H$ is the maximum water depth and $k$ is the roughness height. The selected roughness configurations are based on laboratory experiments, which are used for validating numerical simulations. Results from the LES, in turn, complement the experiments in order to investigate the time-averaged flow properties at much higher spatial resolution. The concept of the double-averaging (DA) of the governing equations is utilized to quantify roughness effects at a range of flow properties. Double-averaged velocity profiles are analysed and the applicability of the logarithmic law for rough-wall flows of intermediate submergence is evaluated. Momentum flux components are quantified and roughness effect on their vertical distribution is assessed using an integral form of the DA-equations. The relative contributions of pressure drag and viscous friction to the overall bed shear stress are also reported.
\end{abstract}

Key words: wall roughness, Large Eddy Simulation, double averaging.

(C) 2008 Institute of Geophysics, Polish Academy of Sciences 


\section{INTRODUCTION}

Nearly all flows in environment and engineering are hydraulically rough, i.e., roughness elements protrude from the viscous sublayer into the turbulent region. Due to their importance and also their complexity, turbulent flows over rough surfaces have been extensively studied in the past (e.g., Perry et al. 1969, 1987, Townsend 1976, Raupach et al. 1991) and continue to be a subject of comprehensive experimental and numerical studies at present (e.g., Leonardi et al. 2003, Jiménez 2004, Stoesser and Rodi 2004). Vertical profiles of velocities and other parameters over rough beds differ from those over a smooth bed since the nature of the total drag is significantly different when roughness elements are present. However, the comprehensive functional relationships between the roughness geometry and the effects of the roughness on the mean flow and turbulence are still to be found.

Most natural water flows and near-bed boundary layers are relatively shallow with respect to the multi-scale bed roughness (e.g., rivers, estuaries, overland flows, marine boundary layers). With no rigorous theory available, however, these low-submergence flows are nearly always studied using the logarithmic boundary layer concept (LBL), which is based on the universal logarithmic velocity distribution and which is justified only for flows with large relative submergence (e.g., Raupach et al. 1991, Jimenez 2004). Indeed, in a recent review Jimenez (2004) pointed out that for the logarithmic layer to occur the flow depth $H$ to the roughness height $k$ ratio should exceed at least 40 . The erroneous assumption of a logarithmic boundary layer for relatively shallow flows (i.e., water-depth-over-roughness-height $H / k<40$ ) may lead to theoretical and experimental misinterpretations in flow dynamics. For instance, the LBL concept is often misused in estimating bed shear stresses in flows with small relative submergence, which are central to analysing bed stability, erosion, re-suspension, and other geomorphological processes. Although applicability of the LBL concept for low-submergence flows has been questioned since the seventies, an alternative theory for these low-submergence flows is still lacking. In recent publications, Nikora et al. $(2007 \mathrm{a}, \mathrm{b})$ promoted the concept of the double-averaged (in time and in planes parallel to the mean bed) hydrodynamic equations and, based on them, identified four distinct flow types depending on their relative submergence (see also, Nikora et al. 2001). Flow type I represents flows with high relative submergence and contains the outer layer, the logarithmic layer, the form-induced (or dispersive) sublayer (below the logarithmic layer and just above the roughness crests, where the time-averaged flow may be influenced by individual roughness elements), and the interfacial sublayer, which occupies the flow region between roughness crests and troughs and where new players in the momentum balance appear: skin friction and form drag. Together, the form-induced and interfacial sublayers form the roughness layer. In the case of permeable beds as in gravel-bed rivers, there is also a subsur- 
face layer below the roughness layer. The other three flow types are: (II) flow with intermediate relative submergence consisting of the subsurface layer (if applicable), a roughness layer, and an upper flow region which does not manifest a genuine universal logarithmic velocity profile as the ratio $H / k$ is not large enough; (III) flow with small relative submergence with a roughness layer extending to the free surface; and (IV) flow over a partiallyinundated rough bed with the interfacial sublayer as the upper (or only) flow region. The above flow subdivision and flow types represent a useful schematization that may help in developing relationships for vertical distribution of flow characteristics. As an example, Nikora et al. (2004) used this schematisation in developing relationships for the vertical distribution of the double-averaged longitudinal velocity $\langle\bar{u}\rangle$ within the interfacial sublayer, which represents the lower and least studied part of the roughness layer.

This paper develops further this line of research and is based on a Large Eddy Simulation (LES) study of turbulent open-channel flow over transverse square bars on a channel bed. Two roughness types are considered, k-type and transitional type between d-type and k-type, as defined by Perry et al. (1969). Both flow scenarios represent flows with intermediate submergence (i.e., flow type II with $H / k<40$ ). The main focus of the paper is on the vertical distribution of flow velocity, streamwise turbulence intensities, and the effect of the roughened bed on the momentum fluxes. In a similar manner as in Nikora et al. (2007a, b) and Coleman et al. (2007), the double-averaged momentum equations in integral form are utilized for quantifying and exploring momentum fluxes. Numerical framework and set-up are given first, followed by the time-averaged flow properties and their comparison with experiments. Then, the double-averaged quantities are presented and discussed.

\section{NUMERICAL FRAMEWORK}

The LES code HYDRO3D originally developed at Bristol University (Stoesser 2002) is used to perform the LES simulations. The code solves the filtered Navier-Stokes equations on a Cartesian, block-structured grid discretised following the finite volume method. A non-staggered grid with Cartesian velocity components is used. Both convective and diffusive fluxes are approximated with central differences of second order accuracy. The SIMPLE algorithm is employed in order to conserve mass and to couple the pressure to the velocity field. Time advancement is achieved by a second order explicit Runge-Kutta scheme. HYDRO3D is parallelised allowing domain decomposition with messaging via MPI. The subgrid stresses of the filtered Navier-Stokes equations are computed using the dynamic approach of Germano et al. (1991). The no-slip boundary condition is available for near wall resolution down to the viscous sublayer as is the wall function 
approach of Shuman. Two free surface conditions are implemented in HYDRO3D: (a) rigid lid and (b) level set method, allowing for free surface movement. In the simulations used in this paper, however, the frictionless rigid lid condition was sufficient as the water surface was expected to be flat and parallel to the flow bed. The right-handed coordinate system is implied throughout the paper, i.e., $x$-axis is oriented along the main flow parallel to the bed ( $u$-velocity component), $y$-axis is oriented to the left bank ( $v$-velocity component), and $z$-axis is pointing towards the water surface ( $w$-velocity component), with the origin at the channel bed.

\section{SIMULATIONS, BOUNDARY CONDITIONS, AND SETUP}

The domain size, boundary conditions, and the Reynolds number of approximately 3000 (based on the roughness height and bulk flow velocity) have been chosen to be identical to laboratory experiments of Polatel (2006), who investigated an open-channel flow over the channel bed roughened with square bars of $1 \times 1 \mathrm{~cm}$ (see Fig. 1 for the sketch and definitions). Two configurations with wavelength-to-height-ratio $\lambda / k=9$ (k-type roughness: 'isolated' roughness elements with $\lambda / k>5$, Perry et al. 1969) and $\lambda / k=4.5$ (transitional between d-type and k-type: wake-interference when $\lambda / k \approx 4-5$ ) have been selected in order to examine roughness effects. The data from Polatel's (2006) experiments are used for validating the LES code and are also combined with simulated data in the analysis of the flow structure. The roughness Reynolds number $R e_{*}$ based on the roughness height $k$ and the bulk shear velocity $u_{*}$ is around 300 for the transitional case and around 550 for the isolated roughness case ensuring fully hydraulically rough-bed conditions for both scenarios. The water depth to roughness height ratio is $H / k=6.5$, indicating relatively shallow flow conditions, i.e., flow type II of intermediate relative submergence. The computational domain spans $6 H$ in streamwise, $\pi H$ in spanwise, and $H$ in vertical directions, respectively. A number of simulations with different grid resolutions were carried out. In this paper, we will only present the results from the finest grids employed consisting of $630 \times 120 \times 100$ grid points for the $\lambda / k=9$ case (see a part of

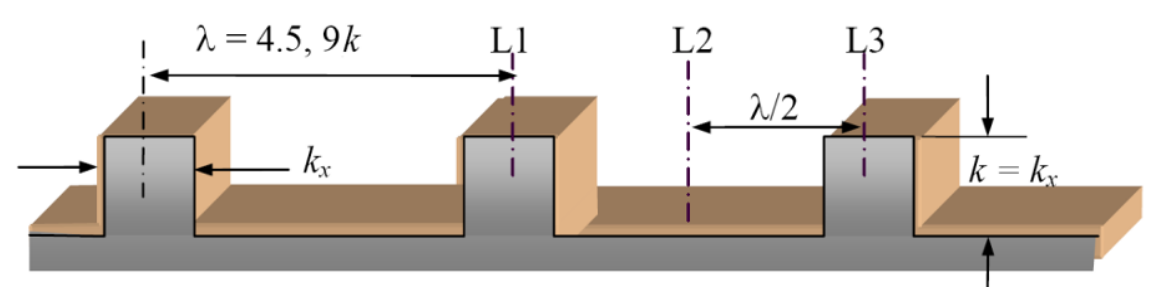

Fig. 1. 2D roughness configuration as investigated by the Large Eddy Simulations. 


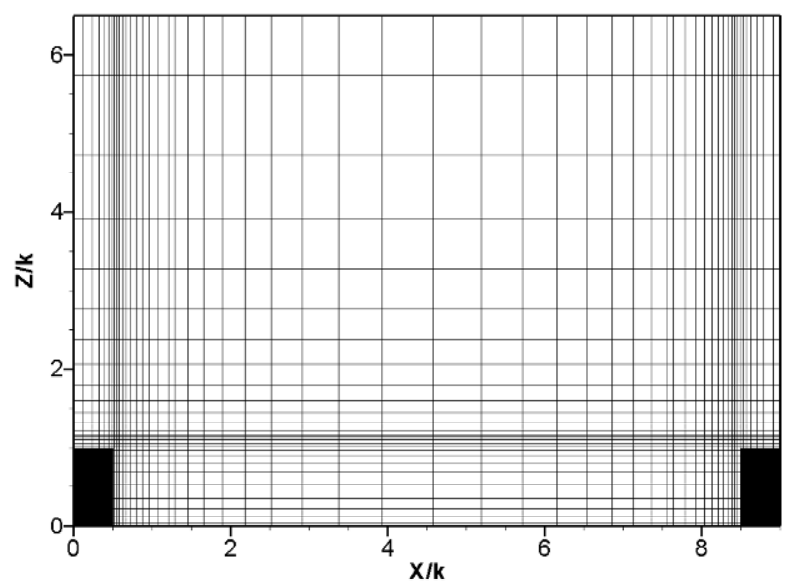

Fig. 2. Longitudinal plane of the grid used for the Large Eddy Simulations (here $\lambda / k=9$, note that only every 5 th grid line is shown).

the grid in Fig. 2, where only every 5 th grid line is shown), and $720 \times 120 \times 100$ for the $\lambda / k=4.5$ case. The grid spacings in terms of wall units were $\Delta y^{+}=\left(\Delta y u_{*} / v\right) \approx 30$ in the spanwise direction and less than 1 for $\Delta x^{+}$and $\Delta z^{+}$near the walls and the roughness elements. Periodic boundary conditions were applied in the streamwise and spanwise directions implying an infinitely long channel. The no-slip boundary condition was used on the surface of roughness elements and on the bed between them. The free surface boundary was treated as a rigid-lid where a slip condition was applied. The key simulation and hydraulic parameters are summarised in Table 1.

The shear velocity and the total wall shear stress used for the normalisation of turbulence quantities and Reynolds stresses were obtained from the time-averaged pressure gradient, i.e., $\tau_{\text {total }}=\left\langle\mathrm{d} p / \mathrm{d} x>H\right.$ and $u_{*}=\left(\tau_{\text {total }} / \rho\right)^{1 / 2}$.

Table 1

Key dimensionless parameters of the Large Eddy Simulations

\begin{tabular}{|l|c|c|}
\hline \multicolumn{1}{|c|}{ Hydraulic parameters } & $\lambda / k=9$ & $\lambda / k=4.5$ \\
\hline Water depth, $H / k$ & 6.5 & 6.5 \\
Bulk velocity, $U_{\text {bulk }} / u_{*}$ & 5.78 & 8.93 \\
Froude number, $F r=U_{\text {bulk }} / \sqrt{g H}$ & 0.36 & 0.5 \\
Reynolds number, $R e_{k}=\left(U_{\text {bulk }} \cdot k\right) / v$ & 2900 & 3200 \\
Roughness Reynolds number, $R e_{*}=\left(u_{*} \cdot k\right) / v$ & 553 & 324 \\
Computational domain: length $\times$ width $\times$ height & $6 H \times \pi h \times H$ & $6 H \times \pi h \times H$ \\
No. grid points: streamwise $\times$ spanwise $\times$ vertical & $630 \times 120 \times 100$ & $720 \times 120 \times 100$ \\
\hline
\end{tabular}




\section{RESULTS AND DISCUSSION}

\section{Time-averaged velocity field}

The time-averaged flow fields above and around roughness elements for both $\lambda / k=4.5$ and $\lambda / k=9$ roughnesses are shown in Fig. 3. A profound disturbance of the flow field in the vicinity of the roughness elements is clearly seen that includes strong separation zones behind the roughness elements and a shear layer above them. Whereas for the flow over isolated roughness elements, $\lambda / k=9$, the mean flow reattaches in the troughs, the time-averaged separation zone for the wake-interference flow, $\lambda / k=4.5$, fully occupies the cavity formed by the roughness elements. In the latter case, the instantaneous flow in the cavity does not contain a stable vortex as is the case of a quasi-smooth flow (d-type), where streamlines connect the tops of the elements, creating a pseudo-wall (Stoesser and Rodi 2004). The timeaveraged flow field undergoes some undulation above the roughness elements, stronger for the isolated roughness elements, $\lambda / k=9$, and weaker for the transitional roughness, $\lambda / k=4.5$. Perry et al. (1969) attributed this roughness-induced streamwise distortion to standing waves, forming just above the roughness elements and occurring in flows over isolated roughness elements, $\lambda / k=9$, and the transitional roughness, $\lambda / k=4-5$. In contrast, Stoesser and Rodi (2004) showed that for the quasi-smooth flow of the dtype, $\lambda / k<4-5$, the flow field above the crests is homogeneous (similar to a flow field above a smooth wall).

Figures 4 and 5 demonstrate the validity of the LES results by comparing the simulated velocity distributions with measurements at two verticals for which point measurements are available. These two locations are L1, which is above the top of a bar, and L2, which is in the centre of the cavity (Fig. 1). For both roughness types, i.e., $\lambda / k=4.5$ and $\lambda / k=9$, the overall agreement of the computed velocity profiles with the measured profiles is encouraging. As can be seen from the profiles at L2, the mean recirculation zone for the
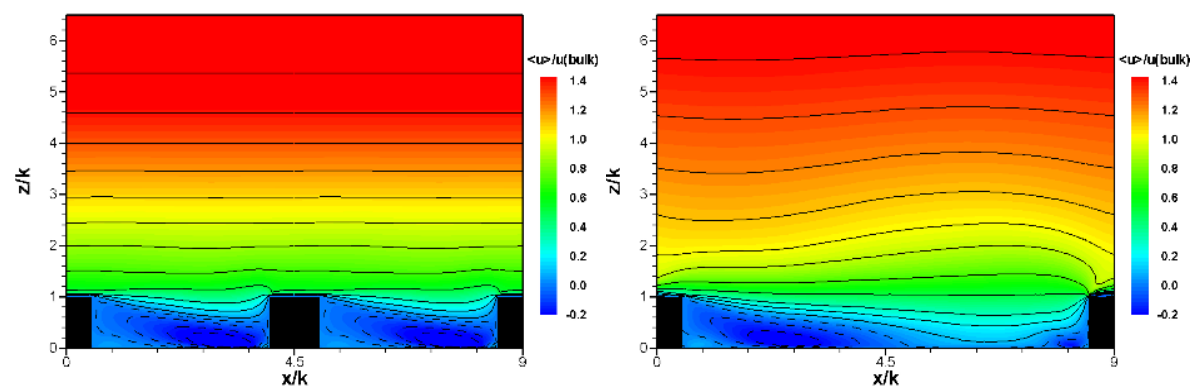

Fig. 3. The time-averaged field of the longitudinal velocity: left: $\lambda / k=4.5$ (transitional roughness), and right: $\lambda / k=9$ (k-type). Dashed lines indicate negative velocities. Local mean velocities are normalised on the bulk flow velocity. See colour version of this figure in electronic edition. 

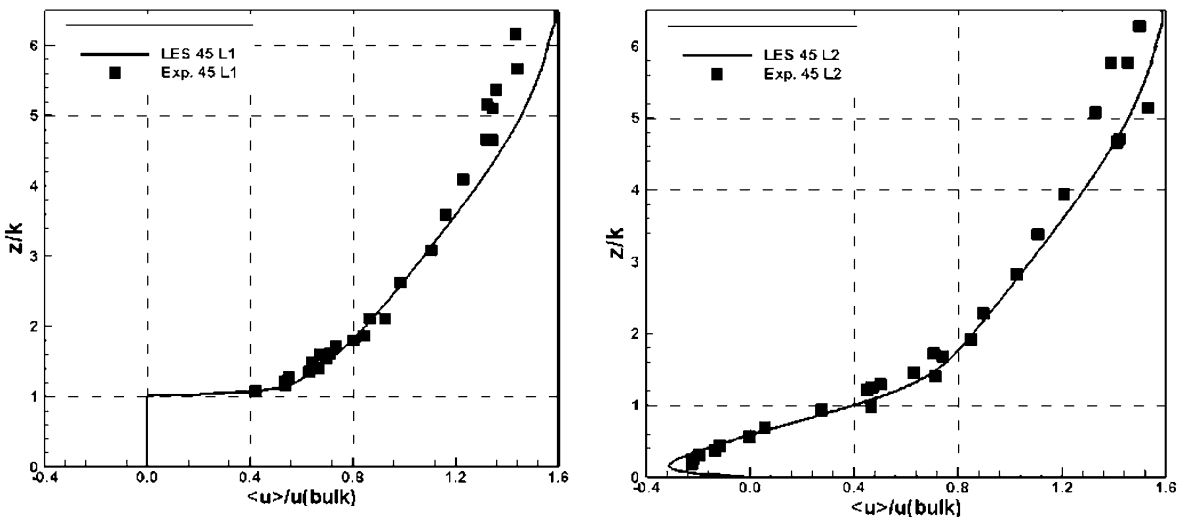

Fig. 4. Time-averaged vertical velocity distribution at two verticals: L1 (left) and L2 (right) for the $\lambda / k=4.5$ case. Local mean velocities are normalised on the bulk flow velocity.
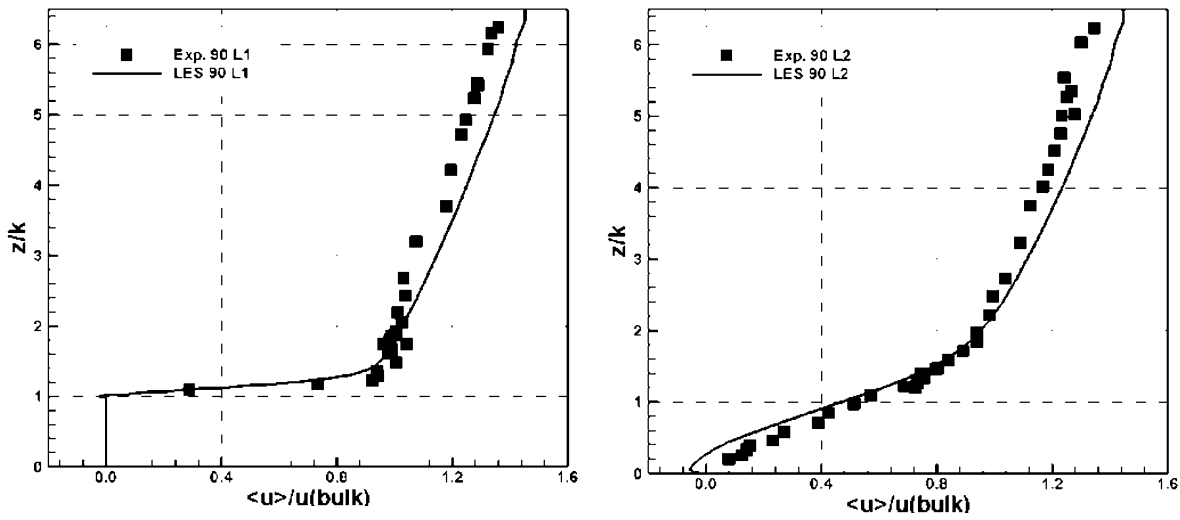

Fig. 5. Time-averaged vertical velocity distribution at two verticals: L1 (left) and L2 (right) for the $\lambda / k=9$ case. Local mean velocities are normalised on the bulk flow velocity.

$\lambda / k=4.5$ case is relatively strong while for the $\lambda / k=9$ case there is (almost) no recirculation midway between two roughness elements suggesting that by this point the flow has recovered. The velocity profiles above the roughness elements at L1 resemble a logarithmic distribution, though there is a tiny recirculation region on the top of the bar for the $\lambda / k=9$ case.

\section{Turbulence intensities and Reynolds stresses}

Figure 6 shows the streamwise relative turbulence intensities within a section of the computational domain. It is noteworthy that for the transitional roughness the maximum streamwise fluctuations occur halfway between the roughness elements whereas for the isolated roughness type they are located 
directly above the top of the elements. In this latter case, most of turbulence is generated when the flow in the trough accelerates towards the downstream bar, causing a small recirculation on the top of the bar. This peak in the vertical $u^{\prime}$ distribution can also be clearly seen in Fig. 8 (left).

In Figs. 7 and 8 the simulated and measured distributions of the streamwise turbulence intensities $u^{\prime}$ normalized by the mean shear velocity $u_{*}$ are compared for both cases, $\lambda / k=4.5$ and $\lambda / k=9$. The match between measured and calculated intensities is fairly good, especially in the near-bed region. There is a significant intensity peak in the vicinity of the roughness tops for both cases. This peak can serve as a potential marker of the upper boundary of the roughness layer (Nikora et al. 2007a, b). Although the maximum values of the normalized turbulence intensities for $\lambda / k=4.5$ and $\lambda k=9$ are similar, they are somewhat smaller than normalized turbulence intensities over smooth walls where $u^{\prime} / u_{*} \approx 3.0$.

Figure 9 presents, for a section of the computational domain, the primary turbulent shear stress $-\overline{u^{\prime} w^{\prime}}$ normalized with the squared shear velocity $u_{*}^{2}=\tau_{\text {total }} / \rho$. The strong shear layer (red area) that forms above the roughness elements is clearly visible and is of a similar size to the roughness layer identified above using maxima in turbulence intensities. Noteworthy, a negative shear stress $-\overline{u^{\prime} w^{\prime}}<0$ occurs in the recirculation zone generated at the leading edge of the bars, being much stronger for the $\lambda / k=9$ case. The negative $-\overline{u^{\prime} w^{\prime}}<0$ is counteracting the negative velocity gradient keeping the turbulent viscosity positive. Figures 10 and 11 present the vertical distributions of the normalized shear stress $-\overline{u^{\prime} w^{\prime}} / u_{*}^{2}$ above the top of the bar and in the centre of the cavity. Again, for both roughnesses $\lambda / k=4.5$ and $\lambda / k=9$ the overall qualitative agreement between measured and calculated values can be observed. The peak in the shear stress in the vicinity of the roughness tops is apparent for both cases. The overall picture for turbulence
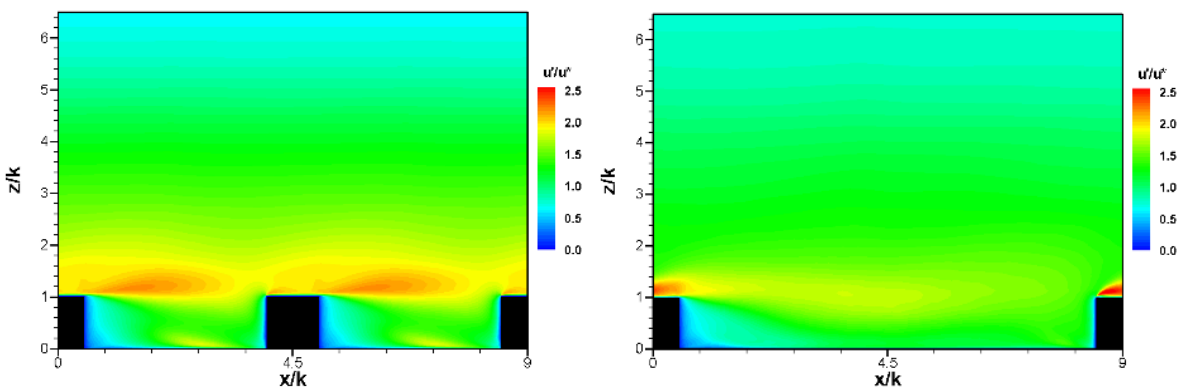

Fig. 6. Distribution of normalized streamwise turbulence intensities $u^{\prime} / u_{*}$ in a longitudinal plane: left: $\lambda / k=4.5$ (transitional roughness), and right: $\lambda / k=9$ (k-type). See colour version of this figure in electronic edition. 

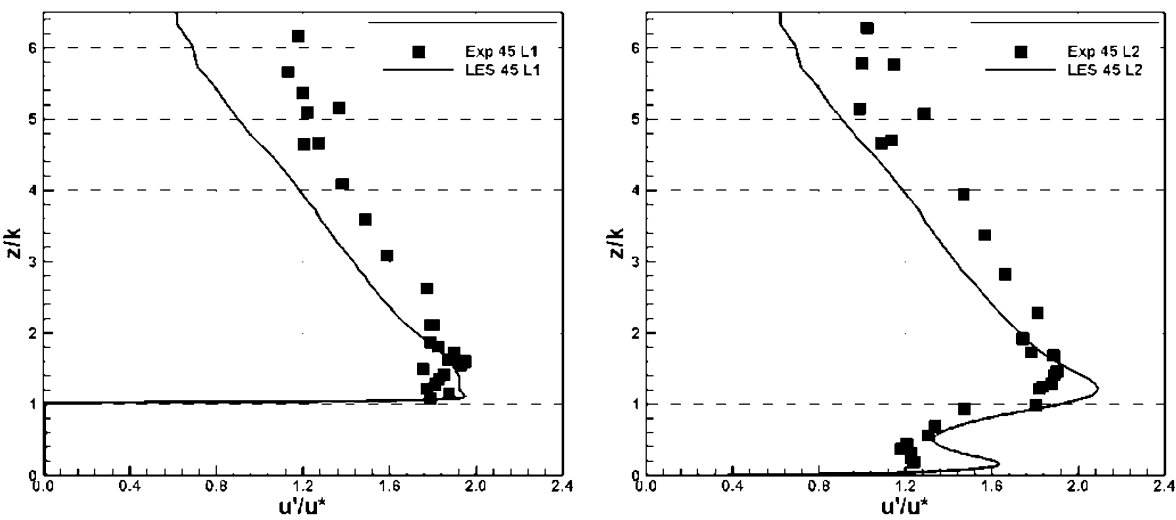

Fig. 7. Vertical distribution of normalized streamwise turbulence intensities $u^{\prime} / u_{*}$ at two verticals: L1 (left) and L2 (right) for the $\lambda / k=4.5$ case.
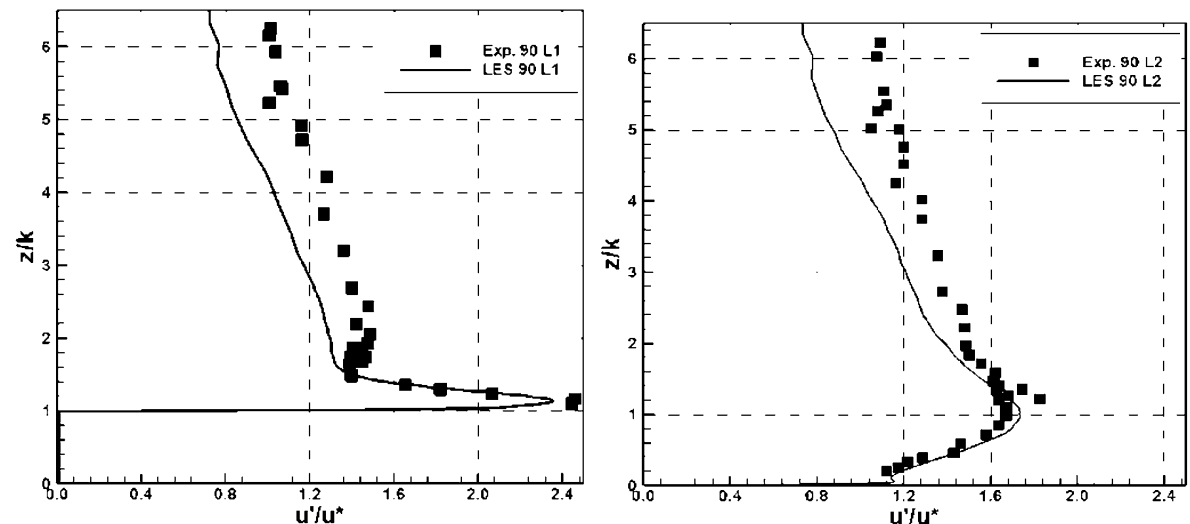

Fig. 8. Vertical distribution of normalized streamwise turbulence intensities $u^{\prime} / u_{*}$ at two verticals: L1 (left) and L2 (right) for the $\lambda / k=9$ case.
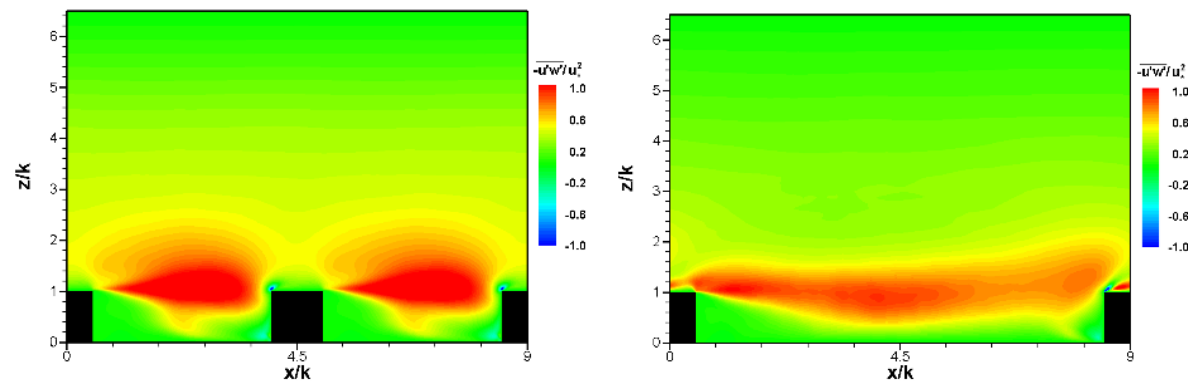

Fig. 9. Turbulent shear stresses $-\overline{u^{\prime} w^{\prime}}$ normalised with $u_{*}^{2}=\left(\tau_{\text {total }} / \rho\right)$ in a longitudinal plane: left: $\lambda / k=4.5$ (transitional roughness), and right: $\lambda / k=9$ (k-type). See colour version of this figure in electronic edition. 

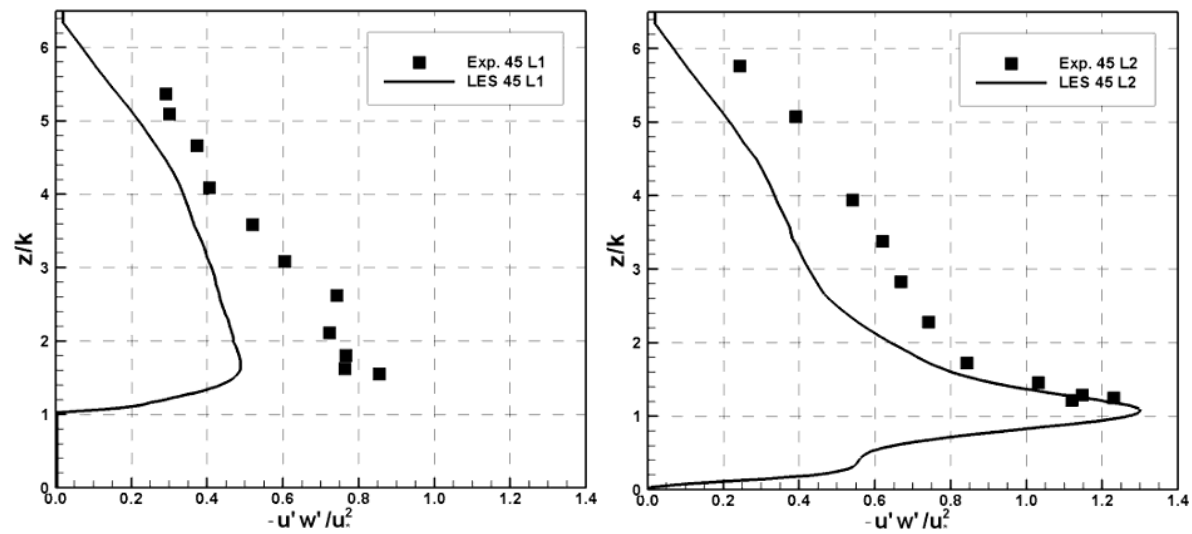

Fig. 10. Vertical distribution of the normalised shear stress $-\overline{u^{\prime} w^{\prime}} / u_{*}^{2}$ at two verticals: L1 (left) and L2 (right) for the $\lambda / k=4.5$ case.
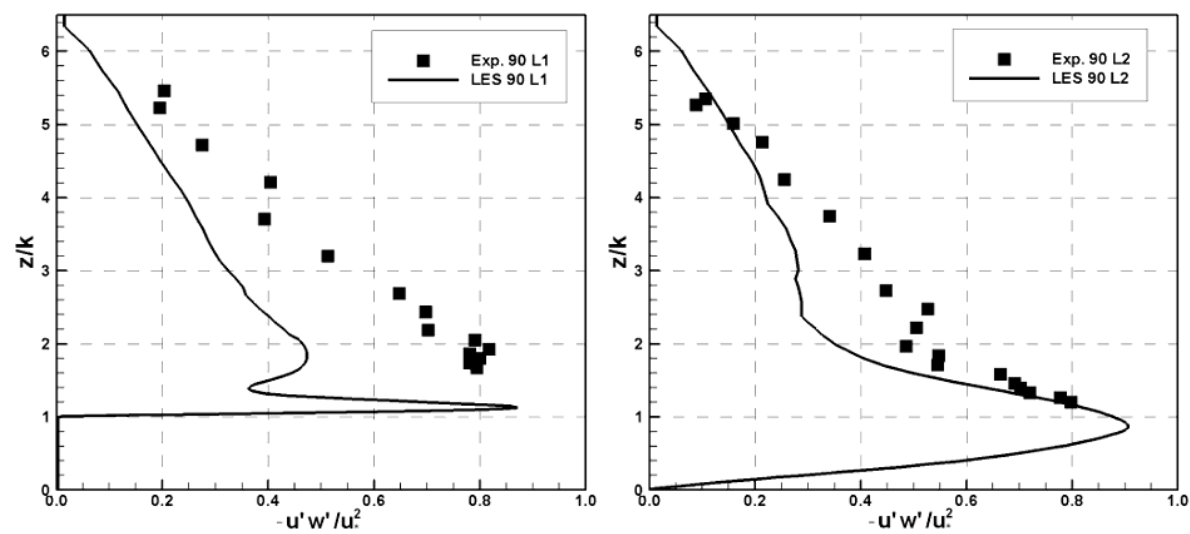

Fig. 11. Vertical distribution of the normalised shear stress $-\overline{u^{\prime} w^{\prime}} / u_{*}^{2}$ at two verticals: L1 (left) and L2 (right) for the $\lambda / k=9$ case.

intensities and shear stresses is consistent with the above men tioned concept of standing waves suggested by Perry et al. (1969). However, quantitative discrepancies in the absolute values of the shear stresses (and also to some extent in the streamwise turbulence intensities) are apparent, particularly along L1, which is a bove the bars. Underestimation of measured stresses might be explained by the fact that LES only captures spatially resolved stresses while the measured stresses over the bars also include numerical subgrid stresses. Further, the use of a rigid lid free surface boundary condition introduces a small continuity defect, which may also lead to an underestimation of local shear stresses and streamwise turbulence intensities. 


\section{Double-averaged quantities, pressure drag, and momentum balance}

Figure 12 shows the double averaged velocity profiles (thick black lines) for $\lambda / k=4.5$ (left) and $\lambda / k=9$ (right), together with the time-averaged velocity profiles along every $20 t h$ vertical gridline along the channel. For the case of the transitional flow $(\lambda / k=4.5$, left), the time-averaged profiles disperse below the roughness tops but above them they closely collapse on a single curve corresponding to the double-averaged profile. In contrast, the dispersion of the time-averaged velocity profiles for the isolated k-type roughness $(\lambda / k=9$, right) extends almost to mid-depth of the flow.
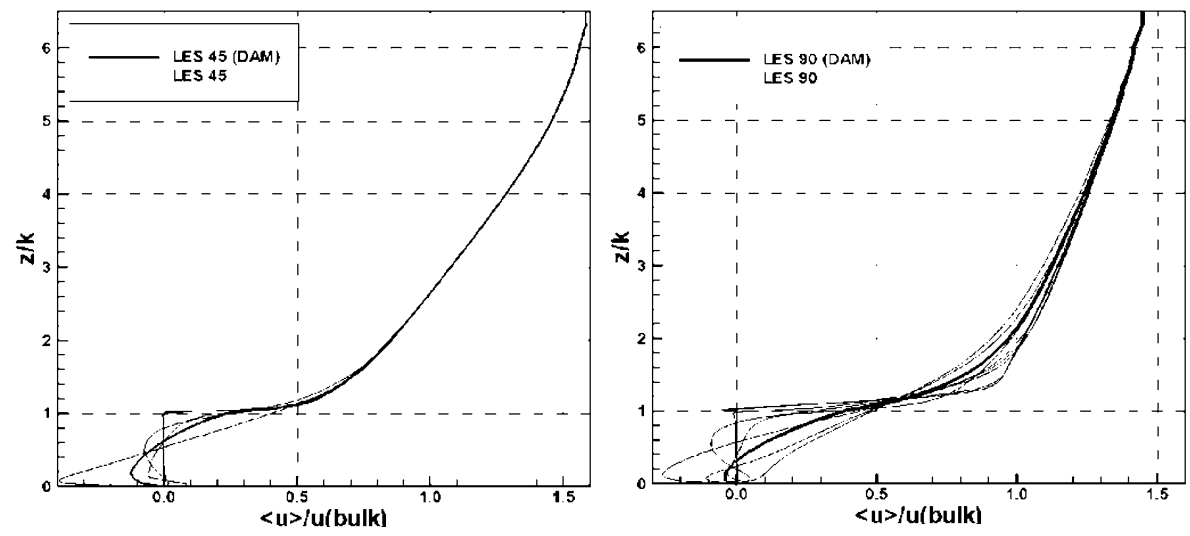

Fig. 12. Vertical distribution of double-averaged streamwise velocity (thick line) together with every 20th LES-time-averaged-data line: left: $\lambda / k=4.5$ (transitional roughness), and right: $\lambda / k=9$ (k-type). Local velocities are normalised on the bulk flow velocity.

Figure 13 presents the double-averaged velocity profiles in semilogarithmic coordinates for both roughness cases together with the respective $\log$ law profiles (calculated as $<U>/ u_{*}=1 / \kappa \ln [(z-d) / k]+B$ ). First of all, the vertical down-shift, i.e., lower relative velocities $\left\langle U>/ u_{*}\right.$, is apparent for the isolated roughness compared to the transitional roughness. This a well known feature for flows over bar-roughened walls (e.g., Leonardi et al. 2003, Coleman et al. 2007) and it reflects the fact that the wall-shear stress increases from transitional roughness to isolated roughness (bef ore it drops again once the bars are moved further apart). In the LES profiles two deviations from the logarithmic distribution are evident for both cases: one is near the roughness elements, indicating a buffer-effect from the roughness, and the other is close to the free surface where the wake law takes over. The nearbed deviation is stronger for isolated roughness reflecting a thicker roughness layer. Not surprising that the wake region is stronger for the $\lambda / k=4.5$ case, counteracting the effect of negative double averaged velocities below the roughness tops. Overall, the agreement between the simulations and the 


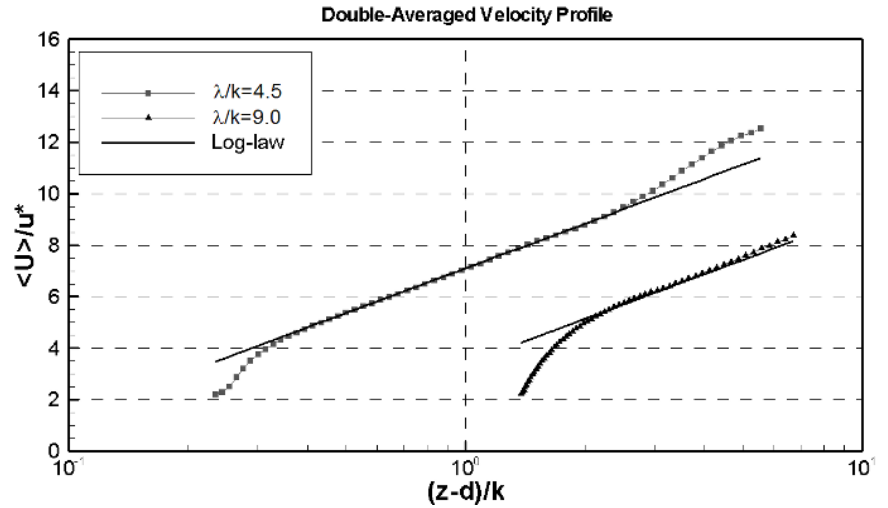

Fig. 13. Double-averaged vertical velocity profile of the two cases together with the respective log-law distributions.

log-law, where the von Karman constant is assumed to be $\kappa=0.4$, is very good, considering that the level of submergence is relatively low. The zeroplane displacement $d$ and the additive constant $B$ in the log law depend on the roughness type (see e.g. Leonardi et al. 2003, Coleman et al. 2007). Using a best fit approach, $d$ and $B$ were evaluated for the two cases and the results are shown in Figs. 14 and 15. Coleman et al. (2007) carried out an extensive experimental study of flow over square bars with varying wavelength-to-height-ratios, and their findings for $d$ and $B$ are included in the figures for comparison. Also included in Fig. 14 is a semi-empirical curve for $B$ from Yaglom (1979). It is interesting to see that the obtained values for $B$ and $d$ agree well with previous studies (e.g., Yaglom 1979, Coleman et al. 2007), though the level of submergence in our study is considerably lower (e.g., $H / k=11$ for the Coleman et al. 2007 study and $H / k=6.5$ here).

Figure 16 presents the double-averaged streamwise turbulence intensity profiles (thick black lines) for $\lambda / k=4.5$ (left) and $\lambda / k=9$ (right), together with the streamwise form-induced (dispersive) intensities (dashed lines).

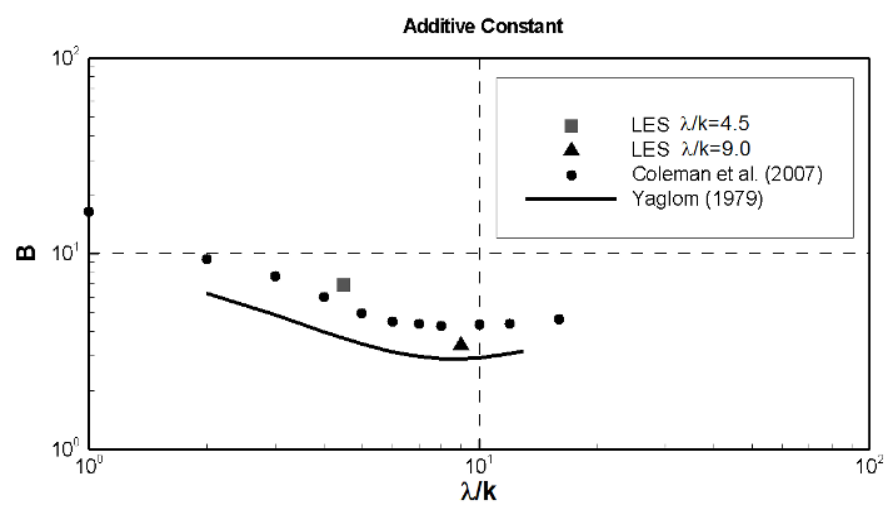

Fig. 14. Additive constant $B$ in the log-law. 


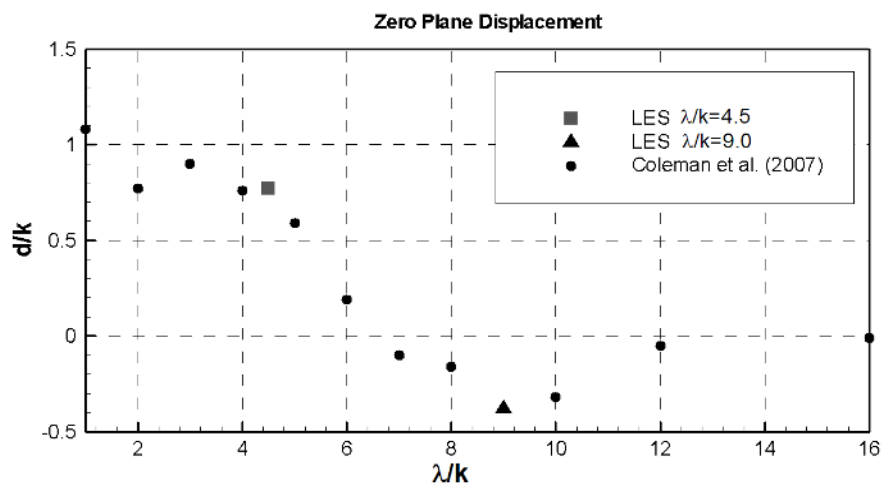

Fig. 15. Normalized zero plane displacement $d$ in the log-law.

Again, a similar trend can be observed as for the velocity distribution in Fig. 12, i.e., the thickness of the near-bed layer where time-averaged profiles deviate from the double-averaged profile is much thinner for $\lambda / k=4.5$ $(z / k \approx 1.5)$ compared to $\lambda / k=9 \quad(z / k \approx 2.5)$. The form-induced intensities are comparable to the shear velocity for both $\lambda / k=4.5$ (left) and $\lambda / k=9$ (right) having maximum values at the roughness tops. Interestingly, combining the normalized turbulence and form-induced intensities together one may obtain values that match the turbulence intensities for smooth-bed flows (where form-induced stresses are absent). This indicates that the roughness layer in a rough-bed flow may be viewed as a counterpart of the viscous and buffer layers in the smooth-bed flow, being responsible for creating shear and turbulence (Nikora et al. 2001). Figures 12 and 15 show that the isolated roughness elements $(\lambda / k=9)$ cause higher turbulence and stronger distortion of the time-averaged and double-averaged velocity fields near the bed and,
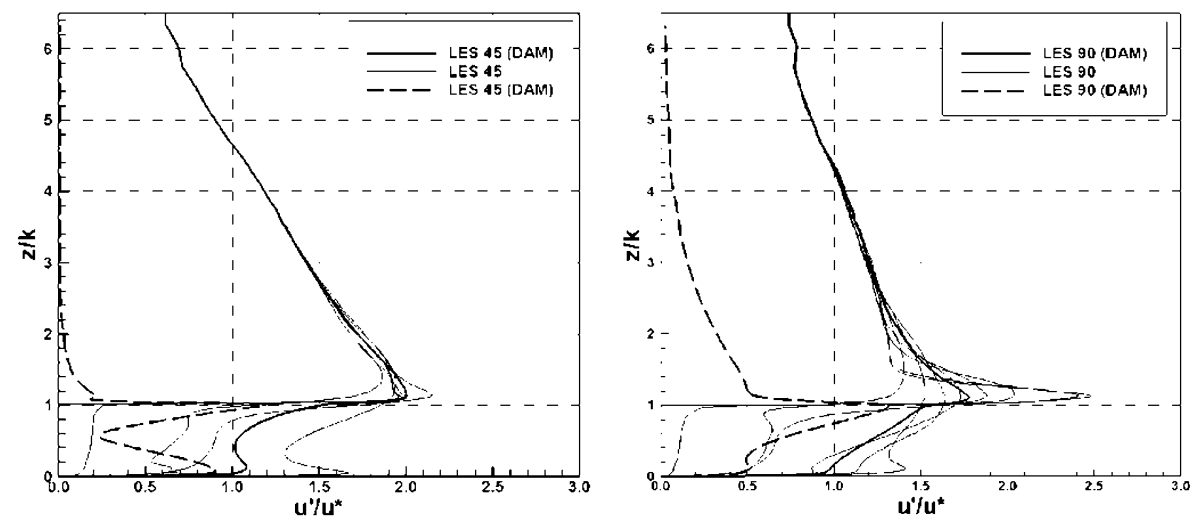

Fig. 16. Double-averaged turbulence intensities $u^{\prime} / u_{*}$ (solid line) together with the vertical distribution of form-induced (dispersive) intensity $\tilde{u} / u_{*}$ (dashed line) and every 30th LES-turbulence intensity-data lines: left: $\lambda / k=4.5$ (transitional roughness), and right: $\lambda / k=9$ (k-type). 
as a result, the roughness layer is thicker for $\lambda / k=9$ compared to that for $\lambda / k=4.5$.

In a two-dimensional flow the vertical distribution of the momentum flux components can be expressed by the following integral form of the momentum equation (Nikora et al. 2007b):

$$
\frac{\mathrm{d} p}{\mathrm{~d} x} \int_{z}^{W S} \phi \mathrm{d} z=-\rho \phi \overline{u^{\prime} w^{\prime}}(z)-\rho \phi \tilde{u} \tilde{w}(z)-\int_{z}^{k} \frac{1}{V_{0}} \iint_{S_{\text {int }}}\left[p n_{1}-v \rho \frac{\mathrm{d} u_{1}}{\mathrm{~d} x_{j}} n_{j}\right] \mathrm{d} S \mathrm{~d} z,
$$

in which the gravity term (left hand side of equation) is balanced by the sum of the turbulent shear stress (first term on right hand side), the form-induced stress (second term on right hand side), and the bed generated flow resistance due to drag and friction (third term on right hand side). For the sake of brevity it is referred to (Nikora et al. 2007b) for details and further explanation.

Vertical distributions of the normalized gravity term (thick black lines), double-averaged Reynolds stress $-\left\langle\overline{u^{\prime} w^{\prime}}\right\rangle$ (curves with triangles), form-induced stress $-<\tilde{u} \tilde{w}>$ (dashed lines with dots), and pressure drag (curves with diamonds) are shown for $\lambda / k=4.5$ (left) and $\lambda / k=9$ (right) in Fig. 17. For both $\lambda / k=4.5$ and $\lambda / k=9$, the spatially-averaged Reynolds stress assumes maximum values just above the roughness tops and then gradually reduce to zero at the water surface and bed. If (quasi)linearity of the $-\left\langle\overline{u^{\prime} w^{\prime}}\right\rangle$ distribution above the roughness tops could be expected, the high degree of linearity of $-\left\langle\overline{u^{\prime} w^{\prime}}>(z)\right.$ below them is noteworthy. The pressure drag dis-
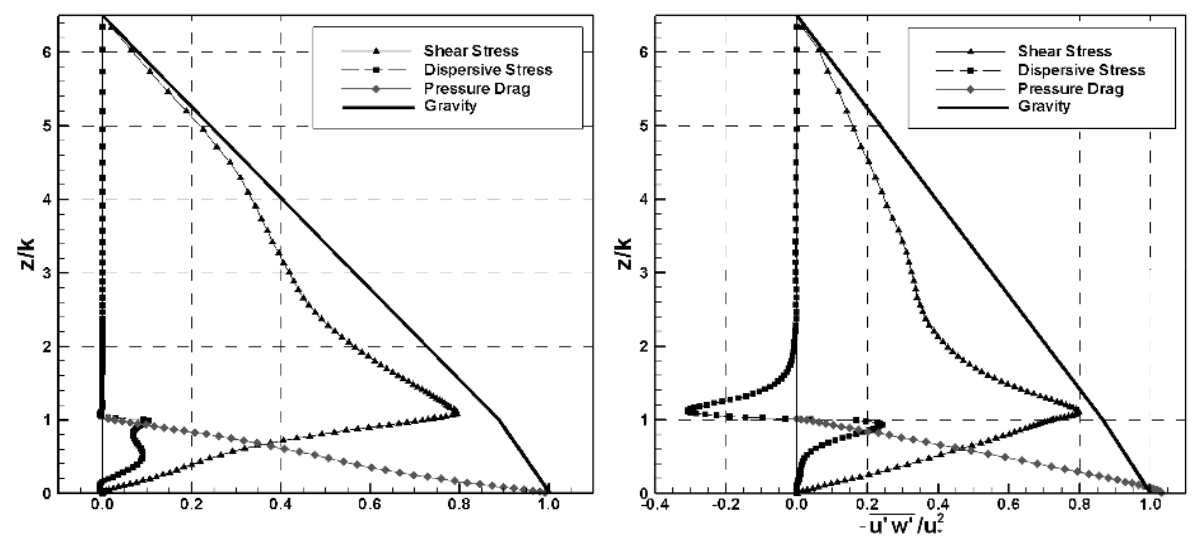

Fig. 17. Spatially-averaged Reynolds shear stress $-\left\langle\overline{u^{\prime} w^{\prime}}>/ u_{*}^{2}\right.$ (solid line) together with the vertical distribution of the form-induced (dispersive) stresses $-<\tilde{u} \tilde{w}>/ u_{*}^{2}$ (dashed line) and the integrated pressure drag: left: $\lambda / k=4.5$ (transitional roughness), and right: $\lambda / k=9$ (k-type). 
tribution is also highly linear changing from zero at the bed to its maximum value at the bar tops. The described features are very similar for both $\lambda / k=4.5$ and $\lambda / k=9$. In contrast, the trends in the distribution of the forminduced stresses $-<\tilde{u} \tilde{w}>$ are significantly different. For the transitional flow $\lambda / k=4.5$, values of $-<\tilde{u} \tilde{w}>$ are almost everywhere positive, except for very thin layers near the bed and the roughness tops where $-<\tilde{u} \tilde{w}>$ adopts very small negative values. Maximum absolute values of $-<\tilde{u} \tilde{w}>$ occur below bar tops where $-<\tilde{u} \tilde{w}>$ reaches $10-12 \%$ of the squared total shear velocity. For the isolated k-type roughness, the distribution of $-<\tilde{u} \tilde{w}>$ is slightly different. They level-off to zero at about $z / k=2$ for the $\lambda / k=9$ case, whereas the dispersive stresses for $\lambda / k=4.5$ show much less variance above the roughness tops. However, the key features of the $\lambda / k=9$ distribution are a negative minimum just above the roughness top and a positive maximum just below it. Both maximums exceed $20 \%$ of the squared shear velocity. The described features in distributions of the double-averaged intensities, pressure drag, and momentum fluxes are consistent with ADV measurements of Coleman et al. (2007), providing at the same time much more detail also being based on much higher degree of averaging.

The distribution of the local wall shear stress (viscous skin friction) along the channel for the two roughnesses is presented in Fig. 18. Peaks in the wall shear stress occur at the leading edges of the bars for both cases. However, for the $\lambda / k=9$ simulation, most of the shear stress on the bar top is negative (indicating the recirculation on the bars), as is in the trough between the bars. In contrast, for the $\lambda / k=4.5$ simulation the shear stress on the bar tops is positive and only a short (though relatively strong) recirculation area is seen. The integrated values for the wall (viscous) shear stress, the integrated pressure drag values and the total drag force resulting from the bed roughness are provided in Table 2. It is noteworthy that in both cases the overall wall friction is negative leading to the total drag being smaller than

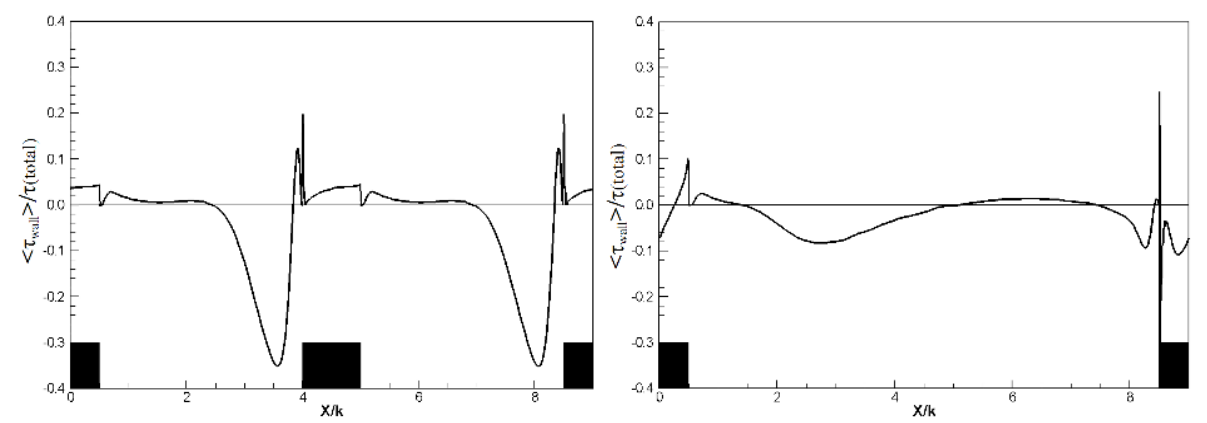

Fig. 18. Longitudinal distribution of the normalized time-averaged shear stress on the wall surface: left: $\lambda / k=4.5$ (transitional roughness), and right: $\lambda / k=9$ (k-type). 
Table 2

Quantification of total, friction and pressure drag for the two cases investigated

\begin{tabular}{|c|c|c|c|}
\hline Case & $\begin{array}{c}\text { Total drag } \\
F_{\text {total }}=\frac{\mathrm{d} p}{\mathrm{~d} x} V_{f}\end{array}$ & $\begin{array}{c}\text { Friction drag } \\
F_{\text {friction }}=\int \mu \frac{\mathrm{d} u}{\mathrm{~d} z} \mathrm{~d} x\end{array}$ & $\begin{array}{c}\text { Pressure drag } \\
F_{\text {pressure }}=\int P \mathrm{~d} z\end{array}$ \\
\hline$\lambda / k=9$ & 12.8 & -0.39 & 13.18 \\
$\lambda / k=4.5$ & 5.85 & -0.01 & 5.86 \\
\hline
\end{tabular}

the overall pressure drag. This result is in qualitative agreement with DNS data of Leonardi et al. (2003).

\section{CONCLUSIONS}

The study reported in this paper has been based on high-resolution LES numerical experiments that match conditions of the laboratory experiments of Polatel (2006) and are close to those of Coleman et al. (2007). Good qualitative and quantitative agreement between simulated and measured flow properties secures confidence in numerical data and supplements laboratory experiments with data of much higher spatial resolution. The simulations also agree very well with DNS data of Leonardi et al. (2003) whose flows were at much lower Reynolds numbers. The main results of this paper can be briefly summarised as follows.

a The time-averaged flow structure for two roughness types with roughness spacings, $\lambda / k=4.5$ and $\lambda / k=9$, is found to be distinctly different although instantaneous and mean separation and recirculation as well as outflow from and inflow into the cavities are shown to occur for both roughness types. Vertical distributions of mean velocities, turbulence intensities, and Reynolds stresses from simulations match time-averaged laboratory data of Polatel (2006). It is found that the distortion of the time-averaged flow field by bed roughness elements is much stronger for $\lambda / k=9$ reaching $z / k \approx 2.5-3.0$, whilst in the case of $\lambda / k=4.5$ the distorted region did not exceed $z / k \approx 1.5$.

- The double-averaged vertical velocity profiles for both roughness types agree well with previous observations and the log-law for rough-bed channel flow. Values for the zero-plane displacement $d$ and the additive constant $B$ in the $\log$-law were obtained from best fits assuming the von Karman constant $\kappa=0.4$. It is found that they strongly depend on the roughness type. Though the relative submergence is lower than in the experiments used for comparisons (Cole- 
man et al. 2007), these values exhibit the same trends, i.e., minima for both $d$ and $B$ are at around $\lambda / k=8-10$.

- The double-averaged flow structure for two spacings, $\lambda / k=4.5$ and $\lambda / k=9$, has some similarities but also differs, with most profound divergence observed near the bed. Among the most interesting findings are (a) quasi-linearity of the double-averaged primary Reynolds stress profile from the bar top towards the bed and water surface (valid for both $\lambda / k=4.5$ and $\lambda / k=9$ ); (b) quasi-linearity of the pressure drag changing from the maximum at the bar top to zero on the bed (valid for both $\lambda / k=4.5$ and $\lambda / k=9$ ); and (c) similar distribution of the form-induced stresses for $\lambda / k=4.5$ and $\lambda / k=9$, however the latter leveling-off to zero further away from the wall. The revealed properties agree well with laboratory experiments of Coleman et al. (2007).

Overall, our findings support the statement of Djenidi et al. (1999) who highlighted inadequacy of a roughness classification scheme based solely on the effect the roughness has on the mean velocity profile. Furthermore, we suggest that the currently used parameters for transverse bar roughness $\lambda / k$ and $H / k$ should be supplemented with $H / \lambda$. Although this parameter is already 'in-built' into combination of $\lambda / k$ and $H / k$, it may still provide a missing dimension in considerations. For example, even at small $\lambda / k<5$ when we could expect 'smooth'-bed d-type flow the situation may depend on $H / \lambda$ and our expectation may not be met. In a similar way as with $\lambda / k$, we can consider three distinct regimes in terms of $H / \lambda: \lambda<<H, \lambda \approx H$, and $\lambda>>H$. These regimes reflect differences in combinations of major turbulence scales $H$ and $\lambda$. The above simplified schematisation suggests that a better way for classification may involve a three-dimensional parametric space $\{\lambda / k ; H / k ; H / \lambda\}$ instead of currently used one-dimensional space $\{\lambda / k\}$.

Acknowledgements. The authors are grateful to C. Polatel for permission to use her experimental data. Two anonymous reviewers provided useful comments that helped to improve the manuscript.

\section{References}

Coleman, S., V. Nikora, S. McLean, and E. Schlicke (2007), Spatially-averaged turbulent flow over square bars, J. Eng. Mech. ASCE 133, 2, 194-204.

Djenidi, L., R. Elavarasan, and R.A. Antonia (1999), The turbulent boundary layer over transverse square cavities, J. Fluid Mech. 395, 271-294.

Germano, M., U. Piomelli, P. Moin, and W.H. Cabot (1991), A dynamic subgridscale eddy viscosity model, Phys. Fluids 3, 1760-1765. 
Jiménez, J. (2004), Turbulent flows over rough walls, Ann. Rev. Fluid Mech. 36, $173-196$

Leonardi, S., P. Orlandi, R. J.Smalley, L. Djenidi, and R.A. Antonia (2003), Direct numerical simulations of turbulent channel flow with transverse square bars on one wall, J. Fluid Mech. 491, 229-238.

Nikora, V., D. Goring, I. McEwan, and G. Griffiths (2001), Spatially-averaged open-channel flow over a rough bed, J. Hydraul. Eng. ASCE 127, 123-133.

Nikora, V., K. Koll, I. McEwan, S. McLean, and A. Dittrich (2004), Velocity distribution in the roughness layer of rough-bed flows, J. Hydraul. Eng, ASCE 130, 1036-1042.

Nikora, V., I. McEwan, S. McLean, S. Coleman, D. Pokrajac, and R. Walters (2007a), Double-averaging concept for rough-bed open-channel and overland flows: Theoretical background, J. Hydraul. Eng. ASCE 133, 873-883.

Nikora, V., I. McEwan, S. McLean, S. Coleman, D. Pokrajac, J. Aberle, L. Campbell, K. Koll, and T.M. Clunie (2007b), Double-averaging concept for rough-bed open-channel and overland flows: Applications, J. Hydraul. Eng. ASCE 133, 884-895.

Perry, A.E., W.H. Schofield, and P.N. Joubert (1969), Rough wall turbulent boundary layers, J. Fluid Mech. 37, 2, 383-413.

Perry, A.E., K.L. Lim, and S.M. Henbest (1987), An experimental study of the turbulence structure in smooth- and rough-wall boundary layers, J. Fluid Mech. 177, 437-466.

Polatel, C. (2006), Large-scale roughness effect on free-surface and bulk flow characteristics in open-channel flows, Ph.D. Thesis, Iowa Institute of Hydraulic Research, The University of Iowa.

Raupach, M.R., R.A. Antonia, and S. Rajagopalan (1991), Rough-wall turbulent boundary layers, Appl. Mech. Rev. ASME 44, 1-25.

Stoesser, T. (2002), Development and validation of a CFD code for open-channel flows, PhD Thesis, Department of Civil Engineering, Bristol University.

Stoesser, T., and W. Rodi (2004), LES of bar and rod roughened channel flow, Proc. Sixth Int. Conf. on Hydro-Science and Engineering, Brisbane, Australia (CD ROM, $12 \mathrm{p})$.

Townsend, A.A. (1976), The Structure of Turbulent Shear Flow, Cambridge University Press, Cambridge, 2nd ed., 429 pp.

Yaglom, A.M. (1979), Similarity laws for constant-pressure and pressure-gradient turbulent wall flows, Ann. Rev. Fluid Mech. 11, 505-540.

Received 29 January 2008

Accepted 30 April 2008 